\title{
磷酸镁/PBS/小麦蛋白复合骨修复材料
}

\author{
王泉翔 ${ }^{1}$, 邬迎阳 ${ }^{1}$, 董谢平 ${ }^{2}$, 马旭辉 ${ }^{3}$, 魏 杰 $^{1}$
}

(1. 华东理工大学生物材料工程研究中心, 上海 $200237 ; 2$. 江西省人民医院 骨科, 南昌 330006; 3. 深圳科聚新材 料有限公司, 深圳 518101)

摘 要: 采用共沉淀法合成磷酸镁, 将磷酸镁(MP)、聚丁二酸丁二醇酯(PBS)和小麦蛋白(WP)进行复合, 制备出 MP/PBS/WP 复合骨修复材料。通过体外降解、生物活性以及细胞培养等实验对复合材料的理化性能及细胞相容性进 行了研究。结果显示: MP/PBS/WP 复合材料在 Tris- $\mathrm{HCl}$ 缓冲液中浸泡 $10 \mathrm{~d}$ 后, 溶液 $\mathrm{pH}$ 从 7.4 上升至 7.51 , 浸泡 $12 \mathrm{w}$ 后，其降解率达到 $58.43 \mathrm{wt} \%$; 复合材料在模拟体液中浸泡 $10 \mathrm{~d}$ 后，其表面形成磷灰石层; 复合材料能促进 MC3T3-E1 细胞的增殖与分化。结果表明: MP/PBS/WP 复合材料具有优良的降解性、生物活性和细胞相容性, 有望成为一种新型 骨修复材料。

关 键 词: 磷酸镁; PBS; 小麦蛋白; 生物复合材料; 骨修复

中图分类号: TB383 文献标识码: A

\section{Magnesium Phosphate/PBS/Wheat Protein Biocomposite for Bone Repair}

\author{
WANG Quan-Xiang ${ }^{1}$, WU Ying-Yang ${ }^{1}$, DONG Xie-Ping ${ }^{2}$, MA Xu-Hui ${ }^{3}$, WEI Jie ${ }^{1}$ \\ (1. Engineering Research Center for Biomedical Materials, East China University of Science and Technology, Shanghai 200237, \\ China; 2. Department of Orthopaedic Surgery, Jiangxi People's Hospital, Nanchang 330006, China; 3. Polymer Science \\ (Shenzhen) New Materials Co., Ltd., Shenzhen 518101, China)
}

\begin{abstract}
Magnesium phosphate was prepared through coprecipitation method, and biocomposite containing magnesium phosphate(MP), polybutylene succinate (PBS) and wheat protein (WP) was fabricated. In vitro degradability, bioactivity and cell responses to MP/PBS/WP composite were investigated. The results showed that the $\mathrm{pH}$ value changed from 7.4 to 7.51 after the MP/PBS/WP composite being soaked in Tris-HCl buffer solution for $10 \mathrm{~d}$. The weight loss of the composite reached $58.43 \mathrm{wt} \%$ after soaking for $12 \mathrm{w}$. Apatite layer could form on the composite surfaces after soaking in SBF solution for $10 \mathrm{~d}$, indicating good bioactivity. In addition, the MP/PBS/WP composite could promote proliferation and differentiation of MC3T3-E1 cells. All data from this study show that the MP/PBS/WP composite has good degradability, bioactivity and cytocompatibility, showing a potential to be used as a new biomaterial for bone repair.
\end{abstract}

Key words: magnesium phosphate, PBS, wheat protein, biocomposite, bone repair

近年来, 镁基生物材料受到广泛关注, 如镁及 合金、含镁生物玻璃和陶瓷、磷酸镁(MP) 等 ${ }^{[1-2]}$ 。 MP 生物材料具有优良的生物相容性、生物活性、
降解性和成骨活性，但其力学强度低，脆性比较大， 成型比较困难 ${ }^{[3-4]}$ 。合成高分子聚丁二酸丁二醇酯 (PBS) 具有良好的生物相容性、降解性、力学性能和

收稿日期：2015-02-02; 收到修改稿日期：2015-05-24

基金项目：国家科技支撑计划(2013BAI05B10); 深圳市战略新兴产业发展专项科资金(CXZZ2012070355537097) National Key Technology R\&D Program of the Ministry of Science and Technology in the 12th Five Year Plan of China (2013BAI05B10); Special Program of Strategic Emerging Industries Development of Shenzhen (CXZZ2012070355537097)

作者简介: 王泉翔(1990-)，男，硕士研究生. E-mail: leo823449871@163.com

通讯作者: 魏 杰, 研究员. E-mail: jiewei7860@sina.com; 董谢平, 教授, 主任医师. E-mail: 13576030901@163.com 
成型加工性，在生物医学领域具有潜在的应用前景， 但其无生物活性 ${ }^{[5-6]}$ 。小麦蛋白(WP) 是一种天然高分 子, 降解比较快, 亲水性也优于合成高分子材料, 但其力学性能较差 ${ }^{[7-8]}$ 。

无机/有机复合材料因其综合了无机物和有 机物各自的优点, 是目前材料科学研究的热点之 - [9]。因此, 在本研究中, 设计了 MP/PBS/WP 三 元复合生物材料, 期望 MP 为复合材料提供生物活 性, PBS 为复合材料提供成型加工性，而小麦蛋白 (WP) 可提高复合材料的降解性。采用溶液浇注法 制备出 $\mathrm{MP} / \mathrm{PBS} / \mathrm{WP}$ 三元复合生物材料, 并研究 其理化性能及对成骨细胞行为(如粘附、增殖和分 化)的影响。

\section{1 实验方法}

\section{1 磷酸镁的合成}

将六水硝酸镁和磷酸氢二铵(摩尔比为 $3: 2$ ) 分 别溶解在蒸馏水中, 用氨水调节两种溶液至 $\mathrm{pH}$ 10。用恒流洜(HL-2S 型, 常州国华仪器有限公司) 以 $100 \mathrm{drop} / \mathrm{min}$ 的滴加速度, 把硝酸镁溶液滴加到 磷酸氢二铵溶液中, 滴加过程中, 持续摚拌溶液, 并用氨水维持体系 $\mathrm{pH}$ 在 10 左右。滴加完成后, 持 续摚拌 $12 \mathrm{~h}$ 。室温下静置 $12 \mathrm{~h}$, 得到白色沉淀, 将 白色沉淀用蒸馏水和无水乙醇洗涤至中性, 用离 心机(TGL-20B 型, 上海安亭科学仪器厂)分离固体 产物, 并在干燥箱(DHG-9023A 型, 上海精宏设备 有限公司)中烘干, 研针研碎, 得到 MP 白色粉末。 所用化学试剂均为分析纯, 购自国药集团化学试 剂有限公司。

\section{2 复合材料的制备}

制备 $\mathrm{MP} / \mathrm{PBS} / \mathrm{WP}$ 复合材料(MP：PBS：WP = $5: 4: 1$, 质量比)。用电子天平 $(\mathrm{AB} 104-\mathrm{N}$ 型, 梅特勒 托利仪器有限公司)将 $2 \mathrm{~g} \mathrm{PBS}\left(\mathrm{MW}=2 \times 10^{4}\right.$, 上海嶅稞 实业有限公司)和 $0.5 \mathrm{~g} \mathrm{WP}$ (分析纯, 东京化成株式会 社)倒入称量瓶中, 加入二甲基甲酰胺(分析纯, 国药 集团化学试剂有限公司), 固液比为 $0.33 \mathrm{~g} / \mathrm{mL}$, 盖上 瓶盖, 置于 $37^{\circ} \mathrm{C}$ 烘箱中。待材料完全溶解后, 取出称量 瓶, 加入 $2.5 \mathrm{~g} \mathrm{MP}$ 粉末, 将整个体系搅拌均匀。将混合 物倒入不锈钢模具中, 使用压片机在 $2 \mathrm{MPa}$ 的压力下 保压 $2 \mathrm{~min}$ 成型, 复合材料被制作成 $\phi 12 \mathrm{~mm} \times 2 \mathrm{~mm}$ 的 圆片。将制备好的圆片样品在蒸馏水中超声清洗 $5 \mathrm{~min}$, 放入 $37^{\circ} \mathrm{C}$ 烘箱干燥 $24 \mathrm{~h}$ 。用同样的方法制备 $\mathrm{MP} / \mathrm{PBS}$ 复合材料(MP : $\mathrm{PBS}=1: 1$, 质量比), 与 PBS 圆片样品作为对照。

\section{3 表征与测试}

\subsection{1透射电镜和 XRD 测试}

采用透射电子显微镜(JEM1400F 型, 日本 JEOL 公司)观察 MP 的形貌; 用 $\mathrm{X}$ 射线衍射仪 (D/max 2550VB/PC 型, 日本 Rigaku 公司)表征各样 品的组成。

\subsection{2 体外降解实验}

记录各样品的初始质量 $W_{0}$, 按照 $0.1 \mathrm{~g} / 20 \mathrm{~mL}$ 的固液比, 将样品放入含有 Tris- $\mathrm{HCl}$ 缓冲液 $(\mathrm{pH}=7.4)$ 的塑料瓶中, 再将塑料瓶放置于恒温振荡箱 (HZP-150 型，上海精宏实验设备有限公司)中振荡 (温度为 $37^{\circ} \mathrm{C}$, 振荡频率为 $120 \mathrm{r} / \mathrm{min}$ )。在特定时间 点, 将样品取出, 用蒸馏水冲洗, 于 $37^{\circ} \mathrm{C}$ 烘箱中干 燥至恒重, 记录样品的质量 $W_{t}$ 。样品的失重率由如 下公式计算:

\section{Weight loss $(\%)=\left[\left(W_{0}-W_{t}\right) / W_{0}\right] \times 100$}

实验过程中, 用 $\mathrm{pH}$ 值测量仪(PHS-3C 型, 中国雷磁 公司)记录不同时间点 Tris- $\mathrm{HCl}$ 缓冲液的 $\mathrm{pH}$ 值, 考 察溶液的 $\mathrm{pH}$ 值随时间而发生的变化。

\subsection{3 体外活性实验}

按照 $0.1 \mathrm{~g} / 20 \mathrm{~mL}$ 的固液比, 将样品浸泡在含有 模拟体液 $(\mathrm{SBF}, \mathrm{pH}=7.4)$ 的塑料瓶中, 置于 $37^{\circ} \mathrm{C}$ 恒温 振荡箱中(振荡频率为 $120 \mathrm{r} / \mathrm{min}$ )。在特定时间点, 将 样品取出, 用蒸馏水冲洗, 置于 $37^{\circ} \mathrm{C}$ 烘箱中烘干。 样品表面的微观形貌和组成用扫描电子显微镜 (SEM，JSM-6360LV 型，日本 JEOL 公司)和 X 射线 能谱(EDS)表征。用等离子体发射光谱仪(IRLS1000 型, 美国热电公司)测试 SBF 中的 $\mathrm{Ca} 、 \mathrm{P}$ 和 $\mathrm{Mg}$ 的离 子浓度(样品浸泡过程中), 考察各离子随时间而发 生的变化。

\subsection{4 细胞实验}

样品用环氧乙烷灭菌, 接种 MC3T3-E1 细胞(中 国科学院细胞库)于样品表面(密度为 $1 \times 10^{4} \mathrm{cell} / \mathrm{mL}$ ), 置于 $37^{\circ} \mathrm{C} 、 95 \%$ 相对湿度、 $5 \% \mathrm{CO}_{2}$ 的培养箱中培养。 $7 \mathrm{~d}$ 后, 用 $2.5 \%$ 的戊二醛固定细胞, 通过 FITC 和 DAPI 对细胞染色, 用激光共聚焦显微镜 (Nikon A1R 型, 日本 Nikon 公司)观察细胞形态和计数。

细胞培养 1、3、7 d 后, 将材料取出, 转入新的 24 孔板中, 加入 $500 \mu \mathrm{L}$ 的 CCK8 试剂(碧云天生物技 术有限公司), 放回培养箱培养 $4 \mathrm{~h}$, 然后吸取 $10 \mu \mathrm{L}$ 液 体至 96 孔板中, 使用酶标仪(SPECTRAMAXPLUS 型, 美国分子仪器公司)于 $490 \mathrm{~nm}$ 处测定光密度, 每组测量 5 个平行样, 通过计算实验组与空白对照 组的光密度比值得到增殖率, 考察细胞在材料表面 的增殖。 
细胞培养 7、 $10 、 14 \mathrm{~d}$ 后, 加入 $500 \mu \mathrm{L}$ 浓度为 $1 \%$ 的乙基苯基聚乙二醇溶液, 获得细胞裂解液, 每 孔加入 $50 \mu \mathrm{L}$ 浓度为 $1 \mathrm{mg} / \mathrm{mL}$ 的 P-硝基苯磷酸盐溶 液, 室温放置 $15 \mathrm{~min}$, 再加入 $100 \mu \mathrm{L}$ 的 $0.1 \mathrm{~mol} / \mathrm{L}$ 的 $\mathrm{NaOH}$ 终止反应。用酶标仪在 $405 \mathrm{~nm}$ 波长处测 $\mathrm{OD}$ 值, OD 值/总蛋白的数据即表示 ALP 活性, 实验以 牛血清白蛋白(BSA)作为标准蛋白, 通过 BSA 蛋白 检测试剂盒测定蛋白浓度。每组测量 5 个平行样, 研 究细胞在材料表面的分化。

\subsection{5 统计方法}

所有数据均通过 Origin 8 软件处理, 以平均值 \pm 标准差的形式表示。 $P>0.05$ 表示实验所获得的数据 无显著性差异, 不具有统计学意义; $P<0.05$ 表示实 验所获得的数据具有显著性差异。

\section{2 结果与讨论}

\section{$2.1 M P$ 的微观形貌及 MP/PBS/WP 复合材料 的 XRD 分析}

图 1(a)是 MP 的透射电镜照片, 由图可见, MP 呈单 层片状, 轻微团聚, 粒径在 100 500 nm。图1(b)是PBS、 $\mathrm{MP} / \mathrm{PBS}$ 复合材料和 MP/PBS/WP 复合材料的外观照片; 由于含有 WP, MP/PBS/WP 复合材料呈淡黄色。
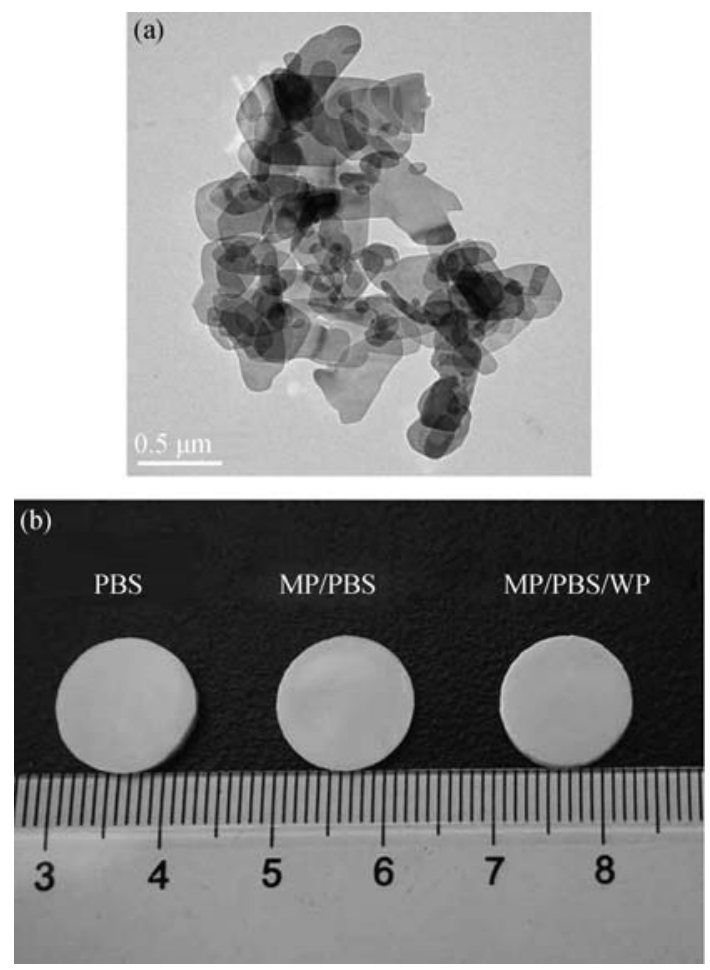

图 1 磷酸镁的透射电镜照片 (a)和 PBS、MP/PBS 复合材料及 $\mathrm{MP} / \mathrm{PBS} / \mathrm{WP}$ 复合材料的宏观照片(b)

Fig. 1 TEM image of magnesium phosphate (a) and photos of PBS, MP/PBS composite and MP/PBS/WP composite (b)
图 2 是 PBS、MP/PBS 复合材料、 MP/PBS/WP 复合材料、 MP 和 WP 的 XRD 图谱。由图可见: 在 $2 \theta=19.3^{\circ}$ 和 $22.2^{\circ}$ 出现的两个峰是 PBS 的特征峰; 在 $2 \theta=13.5^{\circ} 、 21.1^{\circ} 、 23.2^{\circ} 、 29.6^{\circ} 、 31.4^{\circ}$ 和 $34.7^{\circ}$ 出现 的各峰是 MP 的特征峰; 而非晶态的 WP 在 $2 \theta=20^{\circ}$ 附近有一个较宽的弱峰。在 MP/PBS 和 MP/PBS/WP 两种复合材料上均可以找到相应材料的特征峰。这 些结果表明，复合材料包含各单一材料，各单一材 料复合后，其物相没有发生明显的改变。

\section{$2.2 \mathrm{MP} / \mathrm{PBS} / \mathrm{WP}$ 复合材料的体外降解行为}

图 3(A)是 PBS、MP/PBS 复合材料和 $\mathrm{MP} / \mathrm{PBS} /$ WP 复合材料在 Tris- $\mathrm{HCl}$ 缓冲液浸泡不同时间后的 失重率变化。结果显示, $12 \mathrm{w}$ 后, PBS 的降解率为 $9.98 \mathrm{wt} \%$, 降解性低; MP/PBS 复合材料的降解率为 $46.95 \mathrm{wt} \%$, 提示由于 PBS 复合 MP，降解性明显提 高; 而 MP/PBS/WP 复合材料的降解率为 $58.43 \mathrm{wt} \%$, 提示由于 PBS 复合了 MP 和 WP, 降解性进一步提 高。结果表明: 加入 WP, 可以明显提高 MP/PBS/WP 复合材料的降解性。

图 3(B)是 PBS、MP/PBS 复合材料和 MP/PBS/ $\mathrm{WP}$ 复合材料在 Tris- $\mathrm{HCl}$ 缓冲液中浸泡不同时间后 溶液的 $\mathrm{pH}$ 值变化。由图可见: $10 \mathrm{~d}$ 后, PBS 组的 $\mathrm{pH}$ 从 7.4 降为 7.32, 这是由于 PBS 的降解所造成。 $\mathrm{MP} / \mathrm{PBS}$ 复合材料组的 $\mathrm{pH}$ 从 7.4 升到 7.59 , 这是由 于 MP 的水解作用导致溶液的 $\mathrm{pH}$ 有所上升。而 $\mathrm{MP} / \mathrm{PBS} / \mathrm{WP}$ 复合材料组的 $\mathrm{pH}$ 从 7.4 升到 7.51 , 最 终 $\mathrm{pH}$ 低于 $\mathrm{MP} / \mathrm{PBS}$ 复合材料组, 这是由于小麦蛋白 的加入，其降解产物氨基酸引起溶液的 $\mathrm{pH}$ 降低。

理想的骨修复材料应具有合适的降解性，降解 过慢或过快都不利于骨组织生长，从而影响骨缺损 的修复 ${ }^{[10-11]}$ 。另外, 材料降解产物对周围微环境 $\mathrm{pH}$ 有重要影响, 过高或过低的 $\mathrm{pH}$ 会引起细胞毒性

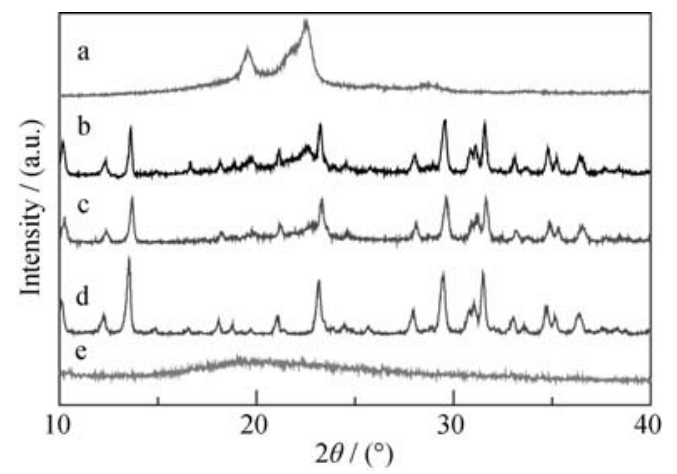

图 $2 \mathrm{PBS}(\mathrm{a}) 、 \mathrm{MP} / \mathrm{PBS}$ 复合材料(b)、MP/PBS/WP 复合材料 (c)、 MP(d) 和 $\mathrm{WP}(\mathrm{e})$ 的 XRD 图谱

Fig. 2 XRD patterns of PBS (a), MP/PBS composite (b), $\mathrm{MP} / \mathrm{PBS} / \mathrm{WP}$ composite (c), MP (d), and WP (e) 

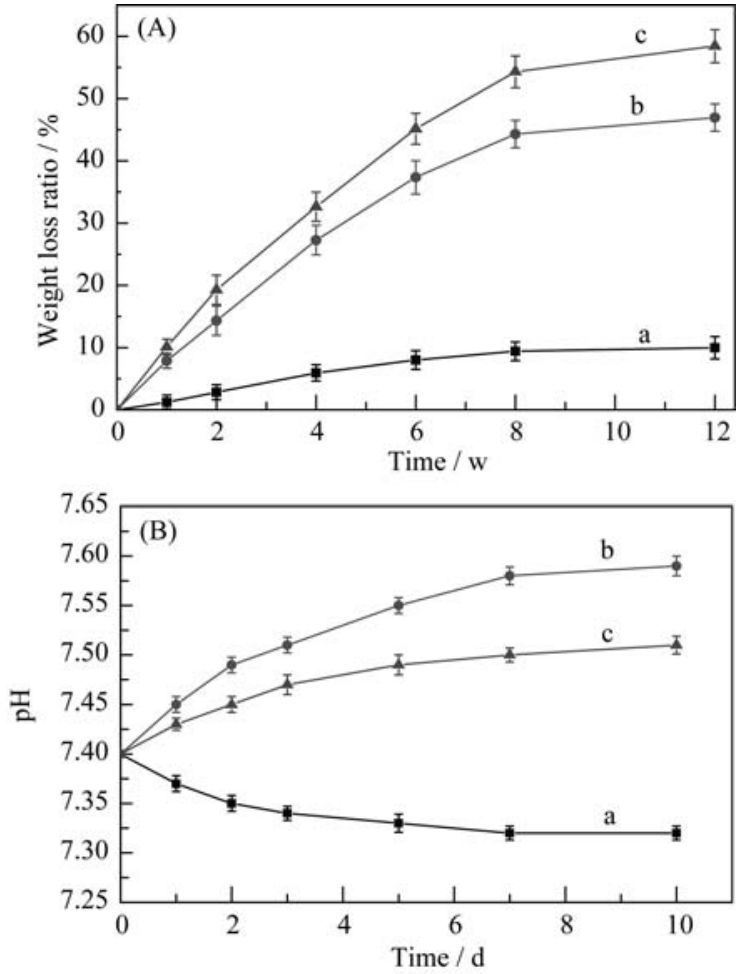

图 3 PBS(a)、MP/PBS 复合材料(b)和 MP/PBS/WP 复合材料(c) 在 Tris- $\mathrm{HCl}$ 缓冲液浸泡不同时间后的失重率(A)和 $\mathrm{pH}$ 变化(B) Fig. 3 Weight loss (A) and $\mathrm{pH}$ change (B) of PBS (a), $\mathrm{MP} / \mathrm{PBS}$ composite (b) and MP/PBS/WP composite (c) soaked in Tris- $\mathrm{HCl}$ solution for different time

反应，从而影响骨的修复效果 ${ }^{[12]}$ 。实验结果表明: $\mathrm{MP} / \mathrm{PBS} / \mathrm{WP}$ 复合材料具有合适的降解性, 降解产 物对溶液的 $\mathrm{pH}$ 值变化没有明显的影响; 而且复合 材料中的 MP 和 WP 可对复合材料降解的微环境中 的酸碱性起微调作用。

\section{$2.3 \mathrm{MP} / \mathrm{PBS} / \mathrm{WP}$ 复合材料的体外矿化行为}

图 4 是 $\mathrm{PBS} 、 \mathrm{MP} / \mathrm{PBS}$ 复合材料和 $\mathrm{MP} / \mathrm{PBS} / \mathrm{WP}$ 复合材料在 SBF 溶液中浸泡 $10 \mathrm{~d}$ 后其表面形貌的扫 描电镜照片。PBS 表面没有磷灰石的生成, 而 $\mathrm{MP} / \mathrm{PBS}$ 复合材料和 MP/PBS/WP 复合材料的表面已 被一层稀疏的磷灰石覆盖, 但 MP/PBS/WP 复合材料 表面的磷灰石生成量多于 MP/PBS 复合材料。结果 提示, PBS 无生物活性，而两种复合材料都具有一定 的生物活性; 其中 $\mathrm{MP} / \mathrm{PBS} / \mathrm{WP}$ 复合材料的生物活性 明显高于 MP/PBS 复合材料; 这是由于 WP 的快速降 解, 使复合材料的比表面积增大, 从而有利于磷灰 石沉积, 生成更多的磷灰石沉积物 ${ }^{[13]}$ 。

图 5 为 $\mathrm{MP} / \mathrm{PBS}$ 复合材料和 $\mathrm{MP} / \mathrm{PBS} / \mathrm{WP}$ 复合 材料在 SBF 溶液中浸泡 $10 \mathrm{~d}$ 的 EDS 图谱。钙、磷 吸收峰的出现表明在复合材料表面形成的是磷灰 石，说明复合材料具有较好的生物活性。此外，
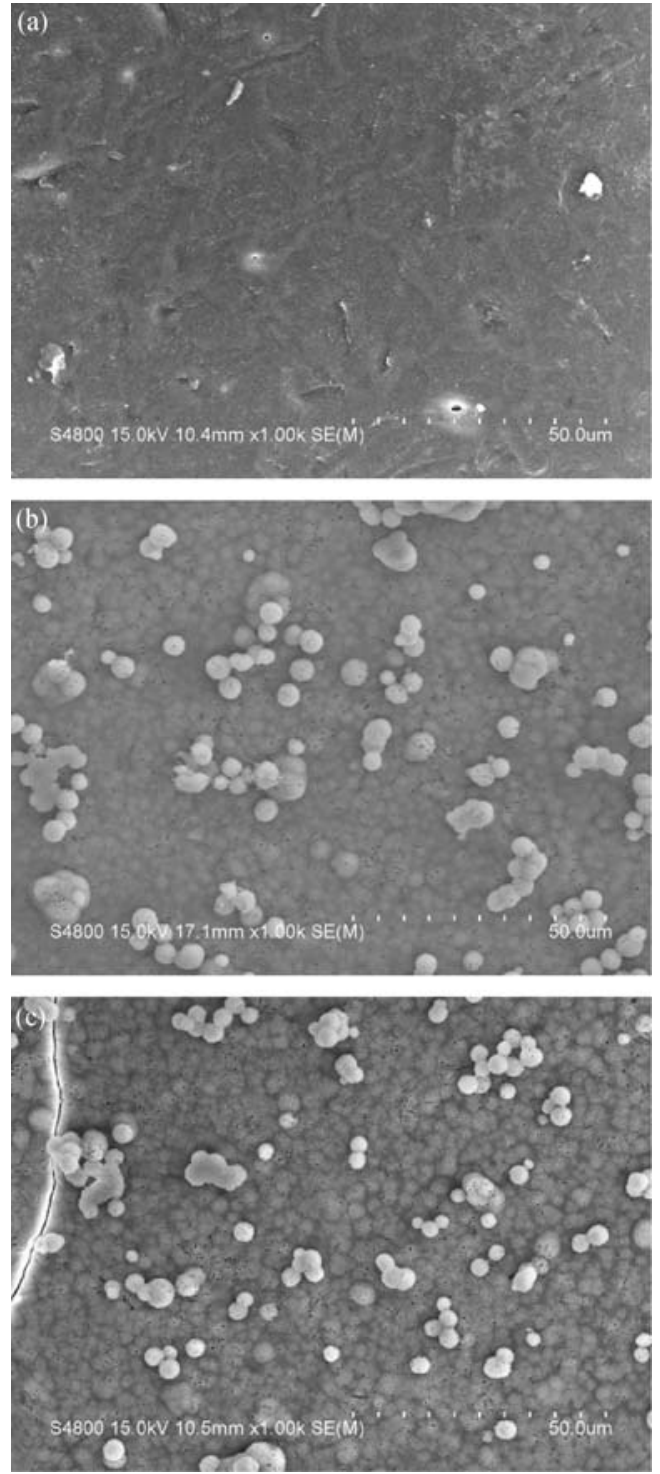

图 $4 \mathrm{PBS}(\mathrm{a}) 、 \mathrm{MP} / \mathrm{PBS}$ 复合材料(b)和 MP/PBS/WP 复合材料 (c)在 SBF 溶液中浸泡 $10 \mathrm{~d}$ 后的扫描电镜照片

Fig. 4 SEM images of surface morphology of PBS (a), MP/PBS composite (b), and MP/PBS/WP composite (c) after soaking in SBF for $10 \mathrm{~d}$

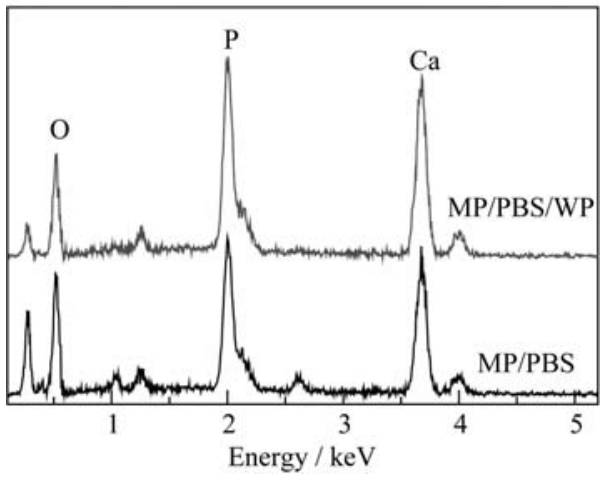

图 $5 \mathrm{MP} / \mathrm{PBS}$ 复合材料和 $\mathrm{MP} / \mathrm{PBS} / \mathrm{WP}$ 复合材料在 $\mathrm{SBF}$ 溶液 中浸泡 $10 \mathrm{~d}$ 后的 EDS 图谱

Fig. 5 EDS spectra of MP/PBS composite and MP/PBS/WP composite after soaking in SBF for $10 \mathrm{~d}$ 
$\mathrm{MP} / \mathrm{PBS} / \mathrm{WP}$ 复合材料表面的钙、磷峰强度略高于 $\mathrm{MP} / \mathrm{PBS}$ 复合材料, 说明 MP/PBS/WP 复合材料表面 的磷灰石含量高于 MP/PBS 复合材料, 这也进一步 表明由于 $\mathrm{WP}$ 的存在, MP/PBS/WP 复合材料比 $\mathrm{MP} / \mathrm{PBS}$ 复合材料具有更好的生物活性。

图 6 是 MP/PBS 复合材料和 MP/PBS/WP 复合 材料在 SBF 溶液中浸泡不同时间后, 溶液中 $\mathrm{Ca}$ 、 $\mathrm{Mg}$ 和 $\mathrm{P}$ 离子浓度的变化情况。由图可见: 两种浸泡 溶液中的 $\mathrm{Ca}$ 离子浓度随时间变化而不断降低, 这 是由于磷灰石沉积, 消耗了溶液中的钙离子; 而两 种浸泡液中的 $\mathrm{Mg}$ 离子浓度随时间变化而不断增加, 这是由于磷酸镁的溶解引起的; 两种浸泡液中的 $\mathrm{P}$ 离子浓度是先降低后升高, 这是由于前期磷灰石沉
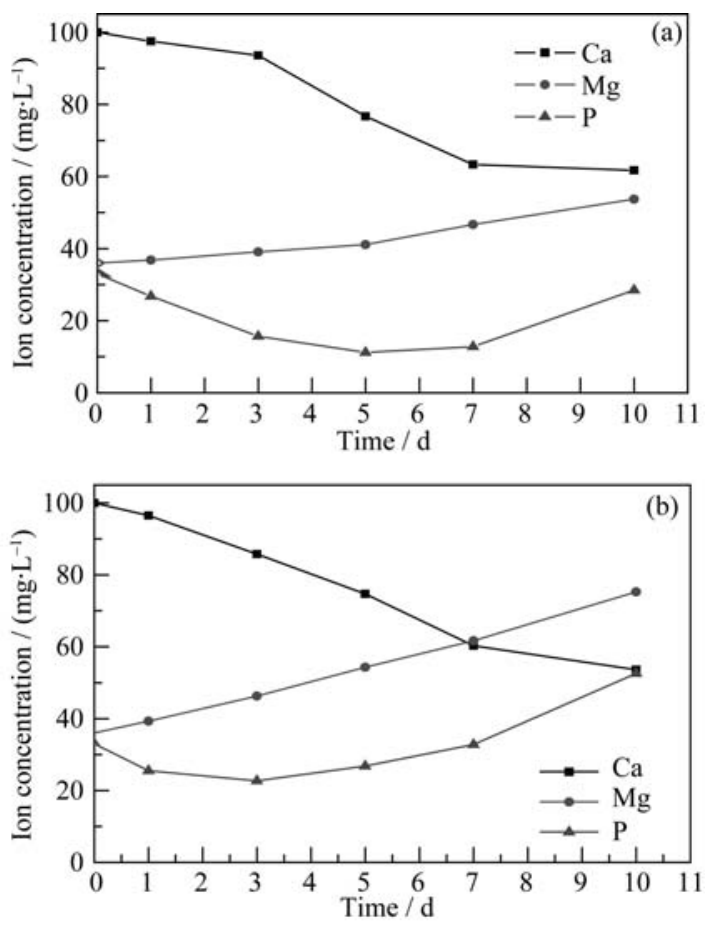

图 $6 \mathrm{MP} / \mathrm{PBS}$ 复合材料(a)和 $\mathrm{MP} / \mathrm{PBS} / \mathrm{WP}$ 复合材料(b)在 $\mathrm{SBF}$ 中浸泡不同时间后, 溶液中的 $\mathrm{Ca} 、 \mathrm{Mg} 、 \mathrm{P}$ 离子浓度的变化

Fig. 6 Ion concentrations changes in SBF after MP/PBS composite (a) and MP/PBS/WP composite (b) immersion for different time
积占主导地位, 消耗了溶液中的 $\mathrm{P}$ 离子, 溶液的 $\mathrm{P}$ 离子降低, 而后期磷酸镁的溶解占主导地位, 使 $\mathrm{P}$ 离子的浓度升高。

此外, 相比 MP/PBS 复合材料, MP/PBS/WP 复 合材料浸泡后, 溶液的离子浓度变化更大, 这是由 于 $\mathrm{MP} / \mathrm{PBS} / \mathrm{WP}$ 复合材料中的 WP 最先降解, 增大了 材料的比表面积, 加快了复合材料中 MP 的降解, 从而有利于磷灰石沉积。将骨修复材料浸泡在模拟 体液中, 考察其体外生物活性是评价和预测材料体 内成骨活性的一个重要指标 ${ }^{[14]}$ 。本实验结果表明, $\mathrm{MP} / \mathrm{PBS} / \mathrm{WP}$ 复合材料具有很好的生物活性。

\section{$2.4 \mathrm{MP} / \mathrm{PBS} / \mathrm{WP}$ 复合材料对细胞增殖与分化 的影响}

图 7 显示, PBS 表面的细胞数量最少, 而 $\mathrm{MP} / \mathrm{PBS} / \mathrm{WP}$ 复合材料表面的细胞数量最多, 铺满 整个材料表面，提示 $\mathrm{MP} / \mathrm{PBS} / \mathrm{WP}$ 复合材料更有利 于细胞粘附、铺展和增殖, 具有优良的细胞相容性, 为该材料进一步的体内植入实验提供了依据 ${ }^{[15]}$ 。

图 8(A)显示细胞在三种材料表面随时间变化而逐 渐增殖。培养 $7 \mathrm{~d}$ 时, 细胞在 MP/PBS/WP 复合份料上 的增殖率明显高于 MP/PBS 复合材料, 而细胞在 PBS 上的增殖率最低。可见, MP/PBS/WP 复合材料能明显 促进细胞增殖，具有良好的细胞相容性。

碱性磷酸酶是成骨细胞分化的标志酶, ALP 的 活性代表成骨细胞分化的程度 ${ }^{[16]}$, 图 8(B) 是 MC3T3-E1 细胞在 PBS、 MP/PBS 复合材料和 $\mathrm{MP} / \mathrm{PBS} / \mathrm{WP}$ 复合材料上培养不同时间的 ALP 活性。 各组 ALP 活性随时间变化而增加。14 d 时, MP/PBS 复合材料和 $\mathrm{MP} / \mathrm{PBS} / \mathrm{WP}$ 复合材料上细胞的 ALP 活 性明显高于 PBS, 说明复合材料能促进细胞分化, 相比于 MP/PBS 复合材料, MP/PBS/WP 复合材料上 细胞的 ALP 活性更高, 说明该复合材料能明显促进 成骨细胞分化。因为 WP 的优先降解, 增加了复合 材料的比表面积, 从而有利于 $\mathrm{Mg}$ 离子的释放, 而 $\mathrm{Mg}$ 离子可促进细胞分化 ${ }^{[17]}$ 。
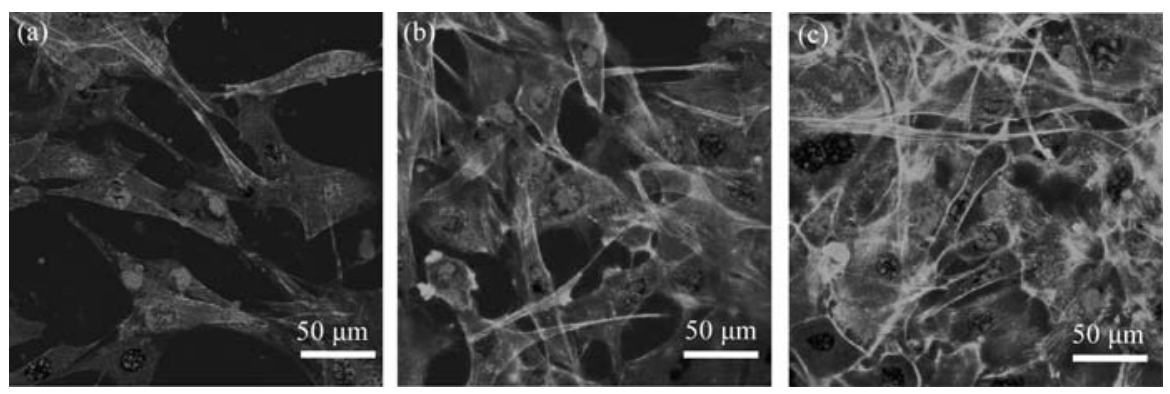

图 7 MC3T3-E1 细胞在 PBS(a)、MP/PBS 复合材料(b) 和 MP/PBS/WP 复合材料(c)上培养 $7 \mathrm{~d}$ 后的激光共聚焦显微照片

Fig. 7 CLSM of cytoskeleton stained by FITC and DAPI after MC3T3-E1 cells cultured on PBS (a), MP/PBS composite (b) and $\mathrm{MP} / \mathrm{PBS} / \mathrm{WP}$ composite (c) for $7 \mathrm{~d}$ 


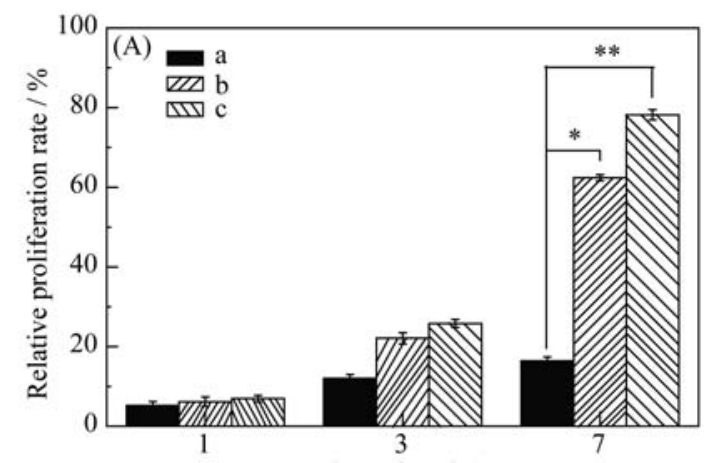

Culture time / d

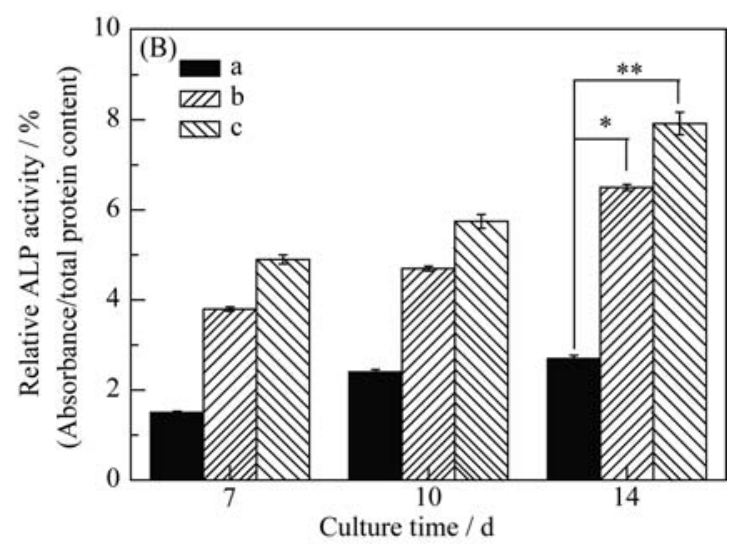

图 $8 \mathrm{MC} 3 \mathrm{~T} 3-\mathrm{E} 1$ 细胞在 $\mathrm{PBS}(\mathrm{a}) 、 \mathrm{MP} / \mathrm{PBS}$ 复合材料(b)、 $\mathrm{MP} / \mathrm{PBS} / \mathrm{WP}$ 复合材料(c)上培养不同时间后的细胞增殖率(A) 和 ALP 活性(B)

Fig. 8 Cell proliferation (A) and ALP activity (B) of MC3T3-E1 cells cultured on PBS (a), MP/PBS composite (b) and MP/PBS/WP composite (c) for different time

\section{3 结论}

采用溶液浇注法制备了磷酸镁/PBS/小麦蛋白复 合骨修复材料。结果显示: 复合材料具有良好的体外 降解性和生物活性; 掺入少量的小麦蛋白可进一步提 高复合材料的降解性和生物活性。细胞实验结果揭示, 磷酸镁/PBS/小麦蛋白复合材料能促进 MC3T3-E1 细 胞粘附、增殖与分化。综上所述，磷酸镁/PBS/小麦蛋 白复合材料具有优良的降解性、生物活性和细胞相容 性，有望作为一种新型的骨修复材料。

\section{参考文献:}

[1] YE X, CAI S, DOU Y, et al. Bioactive glass-ceramic coating for enhancing the in vitro corrosion resistance of biodegradable $\mathrm{Mg}$ alloy. Applied Surface Science, 2012, 259: 799-805.

[2] DU R L, CHANG J. Preparation and characterization of $\mathrm{Zn}$ and Mg doped bioactive glasses. Journal of Inorganic Material, 2004, 19(6): $1353-1358$.

[3] WU F, WEI J, GUO H, et al. Self-setting bioactive calciummagnesium phosphate cement with high strength and de gradability for bone regeneration. Acta Biomaterialia, 2008, 4(6): 1873-1884.

[4] LIU Z S, LIU C S. Progress in research on magnesium phosphate cement as inorganic binder for bone repair. Materials Review, 2000, 14(5): 29-32.

[5] JBILOU F, JOLY C, GALLAND S, et al. Biodegradation study of plasticised corn flour/poly(butylene succinate-co-butylene adipate) blends. Polymer Testing, 2013, 32(8): 1565-1575.

[6] DOREZ G, TAGUET A, FERRY L, et al. Phosphorous compounds as flame retardants for polybutylene succinate/flax biocomposite: additive versus reactive route. Polymer Degradation and Stability, 2014, 201: 152-159.

[7] XU H L, CAI S B, SELLERS A, et al. Electrospun ultrafine fibrous wheat glutenin scaffolds with three-dimensionally random organization and water stability for soft tissue engineering. Journal of Biotechnology, 2014, 184: 179-186.

[8] GÓMEZ M D, PARTAL P, MARTÍNEZ I, et al. Rheological behaviour and physical properties of controlled-release gluten-based bioplastics. Bioresource Technology, 2009, 100(5): 1828-1832.

[9] REZWAN K, CHEN Q Z, BLAKER J J, et al. Biodegradable and bioactive porous polymer/inorganic composite scaffolds for bone tissue engineering. Biomaterials, 2006, 27: 3413-3431.

[10] LIU X, RAHAMAN M N, HILMAS G E, et al. Mechanical properties of bioactive glass(13-93) scaffolds fabricated by robotic deposition for structural bone repair. Acta Biomaterialia, 2013, 9(6): 7025-7034.

[11] CHEN S H, LEI M, XIE X H, et al. PLGA/TCP composite scaffold incorporating bioactive phytomolecule icartin for enhancement of bone defect repair in rabbits. Acta Biomaterialia, 2013, 9(5): 6711-6722.

[12] VELARD F, BRAUX J, AMEDEE J, et al. Inflammatory cell response to calcium phosphate biomaterial particles: an overview. Acta Biomaterialia, 2013, 9(2): 4956-4963.

[13] ZHONG K, VASUDEVAN T V, Somasundaran P. Floatability of apatites of different type and origin: role of surface area and porosity. International Journal of Minerral Processing, 1993, 38(3/4): 177-188.

[14] HUTMACHER D W. Scaffolds in tissue engineering bone and carilage. Biomaterials, 2000, 21(24): 2529-2543.

[15] ELLI L, DOLFINI E, BARDELLA M T. Gliadin cytotoxicity and in vitro cell cultures. Toxicology Letters, 2003, 146(1): 1-8.

[16] QIAO Y Q, ZHANG W J, TIAN P, et al. Stimulation of bone growth following zinc incorporation into biomaterials. Biomaterials, 2014, 35(25): 6882-6897.

[17] ZHANG T, WU X N, HUANG H Y, et al. The beneficial influence of microarc oxidation-coated magnesium alloy on the adhesion, proliferation and osteogenic differentiation of bone marrow stromal cells. Materials Letters, 2014, 137: 362-365. 